\title{
Lossless Currents at High Temperatures
}

\author{
J. Mannhart ${ }^{1}$ D . D. Braak ${ }^{1}$
}

Received: 25 June 2018 / Accepted: 16 August 2018 / Published online: 14 September 2018

(C) The Author(s) 2018

\begin{abstract}
Is superconductivity necessary for zero-resistance currents? We investigate the possibility of designing wires with nonsuperconducting, but nevertheless dissipation-free current flow at room temperature. Guided by a set of constraints for states carrying currents in thermodynamic equilibrium, we suggest using quantum mechanical interference and collapse processes to produce devices that yield the desired function. Two proposals for such devices are presented as examples and their function is discussed also with respect to the second law of thermodynamics.
\end{abstract}

Keywords Lossless currents $\cdot$ Second law $\cdot$ Maxwell's demon $\cdot$ Entropy

Ripple in still water

When there is no pebble tossed

Nor wind to blow

Robert Hunter, Jerry Garcia

\section{Introduction and General Considerations}

The virtually dissipation-free flow of electric currents is a hallmark of superconductivity. High-density supercurrents that are robust to large magnetic fields are the basis for mainstream applications of superconductors, such as superconducting power lines, motors, generators, magnets, and faultcurrent limiters. Great efforts are being made to search for room-temperature superconductors that yield the benefits of these applications without the need for cooling. With the discovery of superconductivity in hydrogen sulfide under extreme pressures [1], the maximum $T_{\mathrm{c}}$ has exceeded $200 \mathrm{~K}$, so that room-temperature superconductivity now seems within reach. The fabrication of useful room-temperature superconducting wires will probably present a further formidable obstacle to be overcome before large-scale applications of superconductors at $300 \mathrm{~K}$ can become reality [2]. In this anniversary issue of the Journal

J. Mannhart

office-mannhart@fkf.mpg.de

1 Max Planck Institute for Solid State Research, 70569 Stuttgart, Germany of Superconductivity and Novel Magnetism, we explore an alternative route to achieve the desired dissipation-free currents. We will submit that lossless current flow at room temperature might indeed be achievable in non-superconducting systems, which we will support by proposing concrete devices. Our discussion is based on refs. [3-5].

Various non-superconducting systems exist that transport electric charge without losses. Atoms, molecules, atomic clusters, and mesoscopically small conducting rings are microscopic examples of such systems (see, e.g., [68]). Dissipation-free charge transport is also possible in macroscopic systems, the salient example being conductors based on the quantum-Hall effect and biased in quantumHall plateaus [9]. However, various difficulties, including the emergence of hot spots at the contacts, have prevented the development of wires based on the quantum-Hall effect. Topologically protected currents in topological insulators have also been proposed as candidates for lossless current flow $[10,11]$. Scattering of the carriers taking place despite topological protection unfortunately obstructs their practical use as dissipation-free conductors.

Systems that carry currents in thermodynamic equilibrium may provide a very different route to dissipation-free currents [3]. In contrast to the topological systems listed above, this approach does not require that scattering be suppressed, which is advantageous for designing long, practical wires. Indeed, in the conductors we propose, inelastic scattering is required to achieve the current flow, as we describe below.

Several partially overlapping restrictions of a fundamental nature may, in principle, prevent a conductor from 


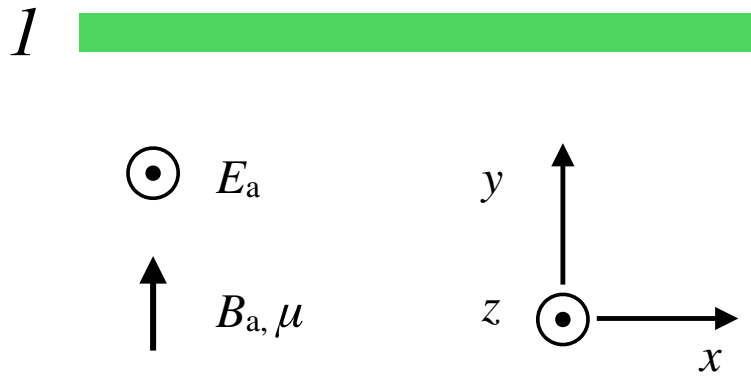

Fig. 1 Sketch of the geometrical configuration. Green $=$ conductor showing the Rashba effect. The conductor with the two contacts 1 and 2 is subject to an electric field $E_{a}$ applied in $z$-direction. An applied magnetic field $B_{a}$ provides spin polarization such that the electron magnetic moments $\mu$ of the conductor point in $y$-direction

carrying a finite current in its ground state [3]. To circumvent these constraints, we must seek conducting systems that

(i) do not have a macroscopically coherent ground state (to avoid superconductivity),

(ii) are not subject to Bloch's second theorem [12, 13],

(iii) break time-reversal symmetry,

(iv) involve processes leading to a non-unitary S-matrix of the complete system, and

(v) are nonreciprocal.

\section{Device Concepts}

It does not seem possible to fulfill requirements (i)-(v) within classical mechanics or by using quantum physics as described by unitary state evolution exclusively. The devices

(a)

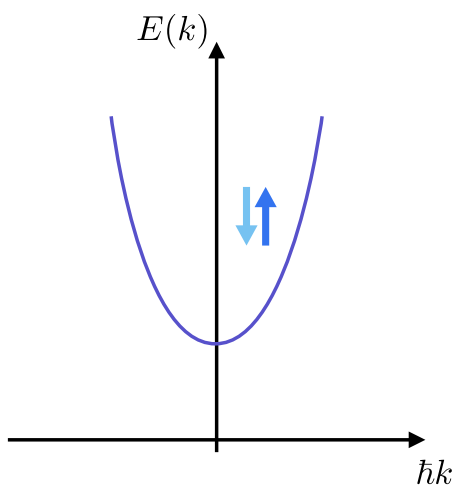

Fig. 2 Possible Influence of an applied magnetic field $B_{a}$ and of the Rashba effect on the band structure of a 1D material. The fields are oriented as sketched in Fig. 1. a Standard parabolic dispersion of the material without applied fields, where $k$ is the wave number in $x$ direction. The blue arrows symbolize the spin directions. b Case where both a large magnetic field $B_{a}$ and an electric field $E_{a}$ are applied. The magnetic field causes a spin splitting of the dispersion, such that two bands offset by twice the Zeeman energy are obtained. The Rashba we propose therefore go beyond classical physics and linear quantum mechanics. By utilizing the generation of coherent quantum states and their collapse due to inelastic interaction, the devices induce the desired nonreciprocal charge current. They thereby use the collapse of the wave function as an integral component. This principle may also be implemented for photonic devices [3-5]. To achieve dissipation-free charge transport, we focus here on electronic devices.

The Rashba effect [14] may serve to achieve nonreciprocal interference of electron waves [3]. In a configuration like the one shown in Fig. 1, the Rashba and Zeeman effects may induce a band structure in a conductor as shown in Fig. 2 (for details, see [3]). In a conductor with such a band structure, electrons moving with the same absolute group velocity $v=\hbar^{-1} \partial E / \partial k$ in $k$ or $-k$ directions are characterized by different de-Broglie wavelengths $\lambda=2 \pi / k$. Combining such a conductor with a standard conductor in a circular or square loop of diameter $2 R$ (Fig. 3), we obtain devices that act on electron waves much as optical isolators act on electromagnetic waves [15] because the traveling waves experience phase shifts depending on the direction of propagation. The loop is designed such that the waves going from port 1 to 2 in the Rashba arm are in phase with respect to the electrons moving in the normal arm, whereas the relative phase of the electrons in the two arms is shifted by $\pi$ if the electrons move from port 2 to 1 .

We start by considering the case that the electrons are not subject to inelastic scattering. An electron injected with some thermal kinetic energy into such an asymmetric Rashba-type quantum ring (Fig. 3) at port 1 to travel to

(b)

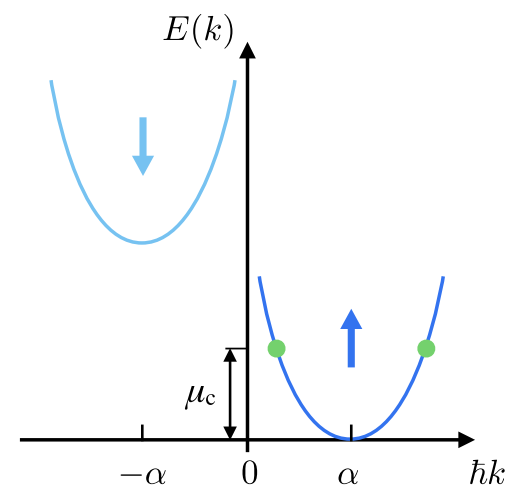

effect induces a shift of the two parabolas along the $k$-axis, such that their minima are positioned at $\hbar k=\alpha$ and $\hbar k=-\alpha$, respectively, where $\alpha$ is the Rashba momentum. The chemical potential $\mu_{c}$ in the device is chosen such that $\mu_{c} \ll \mu B_{a}$. States at the chemical potential (green dots) move in opposite direction with the same absolute value of the group velocity, but are characterized by different de-Broglie wavelengths $\lambda=2 \pi / k$ (after [3]) 


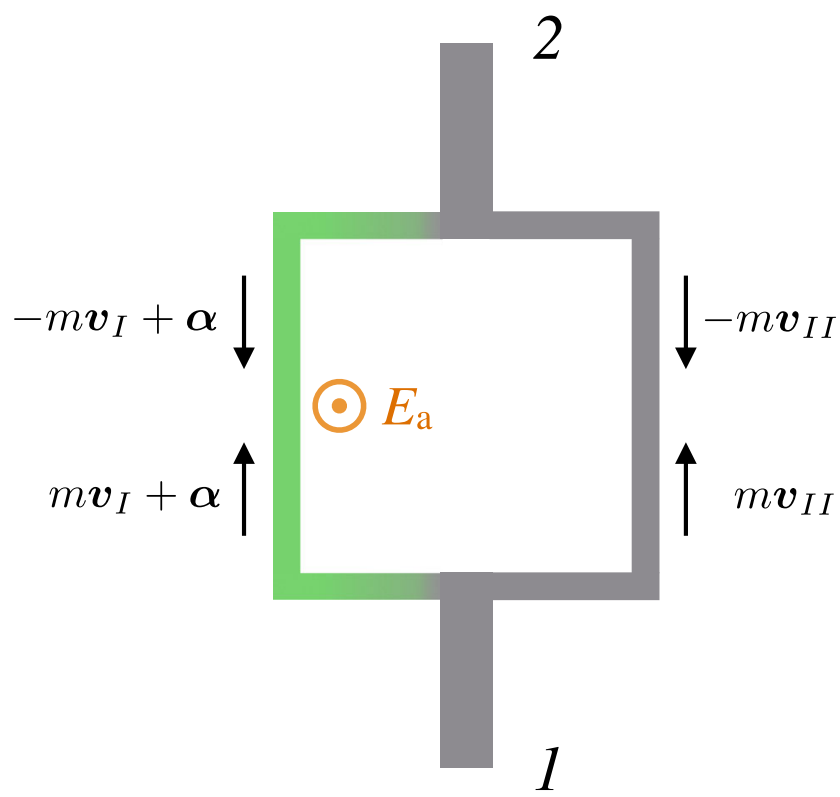

Fig. 3 Quantum loop with non-reciprocal transport based on the Rashba effect. The loop is contacted by the two ports 1 and 2 and is characterized by an inelastic scattering length comparable to its circumference. The loop consists of two different materials. Material I (green): conductor showing the Rashba effect as illustrated in Figs. 1 and 2. Material II (gray): standard conductor with electron states at the chemical potential characterized by a group velocity that differs from that of material I. The momenta listed characterize the electron phase shift per unit length, where $\alpha$ is the Rashba momentum, $v_{i}$ the group velocity of electrons at the chemical potential of material $i$, and $m$ the electron mass (from [3])

port 2 is described by a de-Broglie wave packet with a spatial extension of less than $R$. At port 1 , the wave packet splits into two parts. These travel on either side of the loop, interfere constructively at port 2 , and leave the device there. However, the two wave packet parts of an electron that travels in reverse direction, from port 2 to 1 , arrive at the T-type junction of port 1 with a phase shift of $\pi$ and therefore cannot leave the ring at port 1 . Instead, they continue to circle the ring and travel back to port 2. On their way to port 2, they are not subject to a shift of their phase difference. Therefore, they meet at port 2 again with a phase difference of $\pi$ and are forced to circle back to port 1. As they move from port 2 to 1 , another phase shift of $\pi$ is induced between these half wave packets. Upon arriving at port 1 , they interfere constructively this time, so that now they leave the device at port 1 .

Such loops transmit electrons in both directions without losses. This is a consequence of the unitarity of the overall S-matrix, which enforces reciprocal transmission in any two-terminal device [16]. Nevertheless, the symmetry of the transport has been broken. Electrons traveling in a circular ring from port 1 to 2 leave the ring after having travelled in it for a distance of $\pi R$; electrons traveling from port 2 to $1 \mathrm{do}$ so after having circled the ring $3 / 2$ times, i.e., after having traveled the triple distance, $3 \pi R$.

Note that these devices fulfill requirements (i)-(iii) because the device size is mesoscopic (i); magnetic fields must be applied (ii, iii); and time reversal invariance is broken by the Rashba and Zeeman effects (iii). We now break the reciprocity of the transport by adding inelastic scattering centers coupled to a thermal bath, thereby breaking the unitarity of the evolution of the electron states (iv) [3]. For simplicity, these scattering centers are presumed to be uniformly distributed along the loop. The electron mean free path for inelastic scattering is chosen to be of the order of $2 \pi R$. Several effects relevant to device operation are associated with inelastic scattering: (a) the electron's wave function loses its phase memory [17], (b) the two coherent packets of the wave function collapse into a new wave packet traveling in only one of the half-circles of the ring, and (c) the electron's kinetic momentum changes.

As the scattering centers are uniformly distributed along the loop, the amount of inelastic scattering to which an electron is subjected depends on its port of entry. An electron entering the device at port 1 has a short path $(\pi R)$ before reaching its possible exit port 2 . An electron entering the device at port 2 , however, would need to travel a distance of $3 \pi R$ before it could leave the device if there were no inelastic scattering. On average, electrons entering the device at port 2 are therefore scattered more frequently than electrons entering at port 1 .

If, due to inelastic scattering, the two-packet wave function of an electron collapses into a one-packet wave function the electron continues to move within the half of the ring into which it collapsed. The electron then reaches the next junction that either lets the electron continue to circle or switches it into the adjoining contact. At such a junction, electrons in collapsed states have a comparable probability of leaving the ring or continuing the circle. For unscattered electrons with two-packet wave functions the phase difference of the two wave packets controls the choice of path. Constructive interference makes the electrons enter the contact, whereas destructive interference makes them continue to circle.

As a result, electrons that have entered the ring at port 1 and have moved with high probability coherently to port 2 interfere there constructively, and hence are likely to leave the ring at port 2. In contrast, electrons that have entered the ring at port 2 , and therefore have been scattered more frequently, are significantly more likely to leave the device through their entrance port.

In essence, the device sorts the electrons. Electrons entering the ring in equal numbers at ports 1 and 2 leave the ring more often through port 2 than through port 1.

Using coherent superposition of wave functions (mostly for electrons moving from port 1 to 2 ) and the collapse or 
reduction of wave functions (mostly for electrons moving from port 2 to 1) makes the transport through the twoterminal ring nonreciprocal (v). The collapse processes break the unitarity of the S-matrix of the complete system. Owing to the broken unitarity, the requirement that transport across two-terminal devices must be reciprocal [16] is not applicable to the devices presented here.

This applies to electrons driven by a bias voltage or a bias current and also to electrons that enter the device in thermal equilibrium driven from the contacts by JohnsonNyquist noise [18, 19]. We conclude that, also for uniform temperature distribution, such a device acts like a pump for electrons, shifting them on average from port 1 to 2 . The device thereby generates a difference in the electrochemical potential between these two ports.

These rings demonstrate that electric charge may be transported at room temperature without losses. The transported charge may consist of those electrons that move through the rings, or they may consist of charge flowing through a different, conventional conductor connected to both ports, which are characterized by the generated potential difference. Such devices seem realizable in principle as practical, macroscopic devices, and work at $300 \mathrm{~K}$ or even above.

Such rings or comparable interference devices can be connected in parallel and in series without requiring phase coherence between different rings. They may, for example, be implemented as molecules, solids with complex lattice structures, or be built in large numbers with integrated circuit technology.

Analogs of such devices can be implemented as photonic devices using shunted circulators [4] for transport with asymmetric coherency, half-transparent mirrors or doubleslits to create interference effects, and particles to create photons in two-wave packets, as illustrated by the example shown in Fig. 4. A theoretical analysis of these optical devices shows a temperature imbalance of two equivalent thermal reservoirs and, as a function of time, a decreasing entropy of the isolated, complete system [5]. This optical system crisply reveals the essence of the underlying mechanism of the devices. Whereas outgoing coherent waves are generically created by small particles (Fig. 4, particle $a$ ), nature does not generically generate incoming coherent waves. Owing to this fundamental asymmetry known as the radiative arrow of time, the photon or particle transfer rates $p_{i, j}$ between the two bodies $i$ and $j$ are nonreciprocal, $p_{i, j} \neq p_{j, i}$ in such a device. The asymmetric quantum rings utilize the same mechanism, which is put into action by the different recurrences of the pairwise-coherent wave packets reaching and leaving the T-type junctions (Fig. 3).

These devices, for the function of which we presume the validity of quantum mechanics including collapse
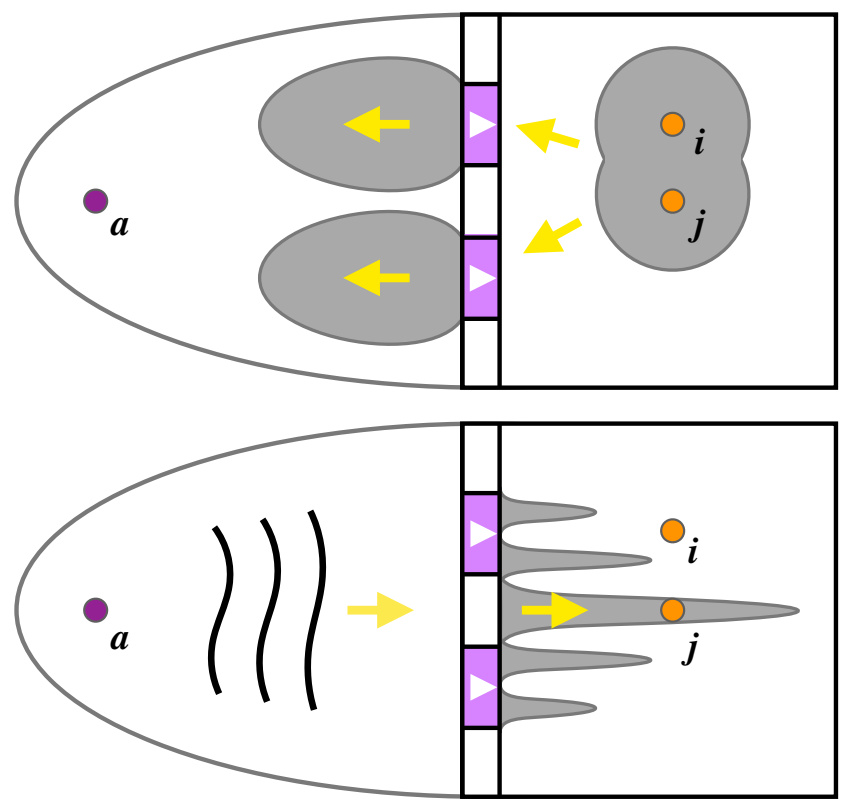

Fig. 4 Sketch of coherent radiation impinging from two sides on two four-port circulators (pink, for details see [4]) arranged in a doubleslit configuration. Ports 1 and 2 of the circulators are oriented toward the left and right cavities, respectively, and the corresponding phasetransmission direction of the circulators is shown by the white arrows. Ports 3 and 4 are terminated by black-body radiators in thermal equilibrium. The walls of the left half of the cavity are provided by mirrors; the walls of the right half are black. Top: two small particles $i$ and $j$ (orange) emit spherical waves that also reach the circulators. The two circulators forward this radiation incoherently into the left cavity. Their radiation characteristics follow Lambert's law as symbolized by the gray radiation plumes and yellow arrows. Bottom: part of the radiation coming from the left is thermally generated by a particle $a$. The coherent radiation of the circulators then emitted to the right generates a double-slit diffraction pattern, causing temperature differences of the particles $i$ and $j$ (orange) that are placed in the maxima and minima of the diffraction pattern, respectively. The device tends to transfer more photons from $i$ to $j$ than vice versa (after [4])

processes, achieve the task of a Maxwell demon [20] and break the second law of thermodynamics [21-23] with the implications this entails (for a more detailed description, see ref. [5]). The devices are closed systems that, starting at a uniform temperature, transform part of their heat energy into another form of energy. In this process, heat flows from the colder particle $i$ to the warmer particle $j$. This energy can consist of the electrochemical potential difference of the ports or their induced temperature difference, which can be converted into electricity by a thermocouple. The nonuniform radiation density (Fig. 4) is not describable using Kirchhoff's law [24] or Planck's radiation law [25]. Also the entropy of the photon gas deviates from the one of a standard black body [26].

For discussions of the possibility of breaking the second law of thermodynamics, see, e.g., [27-30]. Examples of devices proposed to break the second law in a quantum 
mechanical framework are presented [31, 32]. Of particular interest is ref. [32], in which a system has been presented and discussed breaking the second law in the quantum limit through many-body entanglement with a thermal bath at temperatures close to absolute zero. In contrast, the devices presented here rely solely on single-particle properties. As long as the inelastic scattering length is comparable to $R$, they function at any temperature.

The key elements required for such devices to break the second law of thermodynamics are the generation of particle states split into multiple wave packets, the quantum mechanical collapse of the multiple-wave packet states, and the sorting of single and multiple-wave packet states by interference. The latter step transfers the coherence properties of the wave packets into a useful output signal. These robust, single-particle processes are scalable, they function in a wide temperature range including high temperatures, are compatible with a standard room-type environment, and can be implemented in a large variety of devices that act on many species of quantized waves, including electromagnetic, particle, and quasiparticle waves.

\section{Summary and Conclusions}

More than 100 years after the discovery of superconductivity, room-temperature superconductivity finally seems within reach. Whether practical superconducting cables operating at room temperature will also be feasible, is more questionable [2]. However, it seems possible to realize practical, macroscopic, non-superconducting devices that support dissipation-free currents in thermodynamic equilibrium. We have presented two such devices as examples. The generation of coherent wave packets, the interference of quantum states, and in a salient manner, the collapse of wave functions, provide the basis for their function. Remarkably, their functions, for which the validity of quantum physics is presumed, are in apparent disagreement with the second law of thermodynamics [33]. As these devices also seem to be compatible with operation at high temperatures, their potential applications possibly far exceed dissipation-free conduction of electrons.

Acknowledgements Open access funding provided by Max Planck Society. The authors gratefully acknowledge outstanding interactions with T. Kopp and very helpful discussions with A. Alavi, J. Annett, E. Benkiser, A. Brataas, H. Boschker, P. Bredol, T. Giamarchi, S. Hellberg, V. Kresin, G. Leuchs, M. Randeria, E.I. Rashba, B. Rodriguez-Lara, A. Schnyder, C. Schön, R. Valenti, and R. Wanke.

Open Access This article is distributed under the terms of the Creative Commons Attribution 4.0 International License (http:// creativecommons.org/licenses/by/4.0/), which permits unrestricted use, distribution, and reproduction in any medium, provided you give appropriate credit to the original author(s) and the source, provide a link to the Creative Commons license, and indicate if changes were made.

\section{References}

1. Drozdov, A.P., Eremets, M.I., Troyan, I.A., Ksenofontov, V., Shylin, S.I.: Nature 525, 73 (2015)

2. Beasley, M.R.: MRS Bull. 36, 597 (2011)

3. Mannhart, J.: J. Supercond. Novel. Magn. 31, 1649 (2018)

4. Mannhart, J., Bredol, P., Braak, D.: Phase filters for a novel kind of asymmetric transport, submitted to Physica E 5(5) (2018)

5. Braak, D., Mannhart, J.: Inconsistency between quantum theory and thermodynamics displayed by a photonic system, in preparation

6. Bloch, F.: Phys. Rev. 137, A787 (1965)

7. Büttiker, M., Imry, Y., Landauer, R.: Phys. Lett. 96A, 365 (1983)

8. Levy, L.P., et al.: Phys. Rev. Lett. 64, 2074 (1990)

9. Klitzing, K.V., Dorda, G., Pepper, M.: Phys. Rev. Lett. 45, 494 (1980)

10. Qi, X.-L., Zhang, S.-C.: Rev. Mod. Phys. 83, 1057 (2011)

11. Zhang, X., Zhang, S.-C.: Proc. SPIE 8373, 837309 (2012)

12. Bohm, D.: Phys. Rev. 75, 502 (1949)

13. Ohashi, Y., Momoi, T.: J. Phys. Soc. Jpn 65, 3254 (1996)

14. Rashba, É.I.: Sov. Phys. Solid State 2, 1109 (1960)

15. Pozar, D.M.: Microwave Engineering, 4th edn. Wiley, New York (2012)

16. Datta, S.: Electronic Transport in Mesoscopic Systems. Cambridge University Press, Cambridge (1995)

17. In such a system, in thermodynamic equilibrium and on statistical average, the phase memory is erased by inelastic scattering induced by a thermal bath without an energy cost. The systems analyzed, e.g., in L. Szilard, Zeit. f. Phys. 53, 840 (1929) and C.H. Bennett, Int. J. Theor. Phys. 21, 905 (1982) differ

18. Johnson, J.: Phys. Rev. 32, 97 (1928)

19. Nyquist, H.: Phys. Rev. 32, 110 (1928)

20. Maxwell, J.C.: Theory of Heat. Longmans, Green, and Co, London (1871)

21. Clausius, R.: Über die bewegende Kraft der Wärme und die Gesetze die sich daraus für die Wärmelehre selbst ableiten lassen, published 1850, in Abhandlungen über die mechanische Wärmetheorie, vol. 1 (Vieweg)

22. Kelvin: On the dynamical theory of heat. Trans. R. Soc. Edinburgh 1851, and Phil. Mag. IV (1852)

23. Planck, M.: Vorlesungen über Thermodynamik. Veith \& Co, Leipzig (1897)

24. Kirchhoff, G.: Ann. Phys. Chem. 109, 275 (1860)

25. Planck, M.: Verhandl. d. Deutsch. Physik. Gesellsch. 2, 237 (1900)

26. Landau, L.D., Lifshitz, E.M.: Statistical Physics. Pergamon, New York (1959)

27. Lieb, E.H., Yngvason, J.: Phys. Today 53(April issue), 32 (2000)

28. Uffink, J.: Stud. Hist. Phil. Mod. Phys. 32, 305 (2001)

29. Capek, V., Sheehan, D.P.: Challenges to the Second Law of Thermodynamics, Theory and Experiment. Springer, Berlin (2005)

30. Merali, Z.: Nature 551, 20 (2017)

31. Capek, V.: Phys. Rev. E 57, 3846 (1998)

32. Nieuwenhuizen, Th.M., Allahverdyan, A.E.: Phys. Rev. E 66, 036102 (2002)

33. Difficulties also arise regarding the zeroth law, because the direction of the heat flow induced by bringing two bodies into contact may depend on the character of the contact 